EDITORIAL

\title{
Immune regulation in the tumor microenvironment and its relevance in cancer therapy
}

(c) The Author(s), under exclusive licence to CSI and USTC 2021

Cellular \& Molecular Immunology (2022) 19:1-2; https://doi.org/ 10.1038/s41423-021-00738-0

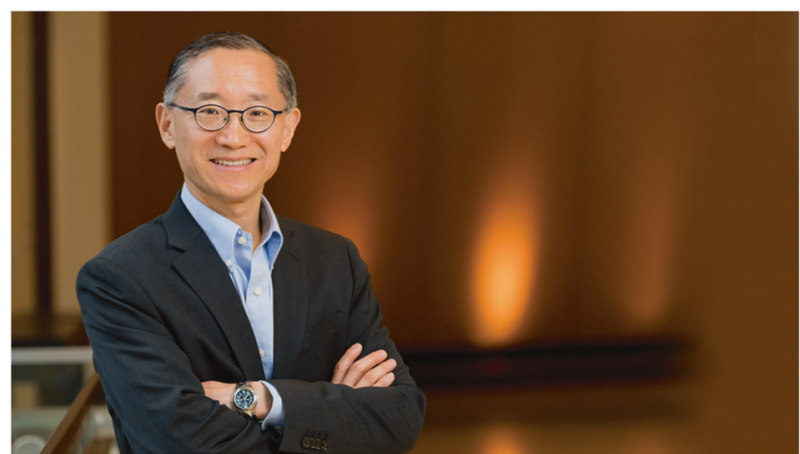

Credit: Dr. Weiping Zou

Cancer immunotherapy, including immune checkpoint blockade and CAR-T cell therapy, has introduced revolutionary approaches to treating patients with cancer. The success and hope of cancer immunotherapy have continuously stimulated high interest in dissecting immunotherapy resistance mechanisms, researching previously unknown therapeutic targets, and informing novel clinical trials in cancer therapy. The notion that the tumor microenvironment holds the key to understanding tumor immunity and developing novel immunotherapy has been well established in the field of tumor immunology and medical oncology [1]. This special issue of Cellular \& Molecular Immunology (CMI) compiles a series of review articles, presenting state-ofthe-art literature and concepts focused on several important aspects concerning the most recent advancement in immune sensitive and resistant mechanisms in the tumor microenvironment, and their relevance in cancer therapy.

Antigen-presenting cells (APCs), such as dendritic cells (DCs) and macrophages, capture, process, and present tumor-associated antigens (TAA), including neoantigens to T cells, resulting in TAAspecific $T$ cell priming and activation. DCs and macrophages express PD-L1 (B7-H1, CD274), contributing in immunotherapyinduced tumor immune responses [2-5].

Given that DCs mediate T cell-cross-priming and activation, it is evident that DCs play a key role in initiating TAA-specific $T$ cell responses, engendering potent antitumor immunity. Following this vein of research, in their review entitled "Dendritic cells in Cancer Immunology", Dr. Murphy discusses DC biology connected with antitumor immune responses. First, they provide a comprehensive picture of recent advances on the development of DC subsets, particularly $\mathrm{CDC1}$ and $\mathrm{CDC} 2$. Second, they cover the cellular and molecular determinants of DC-mediated cross-presentation, and its relevance in cancer immunotherapy. Lastly, they review the longstanding field of $\mathrm{CD}^{+}{ }^{+} \mathrm{T}$ cell "help" and "DC licensing". They have particularly discussed the importance of the CD40-CD40L pathway in $\mathrm{CD}^{+}{ }^{+} \mathrm{T}$ cell help, in which $\mathrm{CD}^{+}{ }^{+} \mathrm{T}$ cells influence the strength or quality of $\mathrm{CD}^{+} \mathrm{T}$ cell responses [6]. Continuing along this line, Dr. Bullock offers a review entitled "CD40 stimulation as a molecular adjuvant for cancer vaccines and other immunotherapies". Dr. Bullock summarizes the rationale and early immunobiology of CD40 as a regulator of DC activation and function. He reviews how CD40 agonists and CD40-based cancer vaccines can be applied in overcoming tumor immune resistance, and combined with immune checkpoint therapy and CAR-T cell therapy [7].

Following the reviews by Drs. Murphy and Bullock, Du et al. systemically examine the IFN $\gamma$ gene signaling pathway in colorectal cancer. The IFNY gene signaling pathway plays a critical role in antitumor immunity. For example, IFNy stimulates expression of MHC-I and MHC-II expression in tumor cells and APCs, including DCs and macrophages, enhancing antitumor immunity. Dr. Du and her colleagues have summarized our current understanding of epigenetic, genetic, and functional integrity of the IFNy signaling pathway in the colorectal cancer microenvironment, and its immunological relevance in therapeutic efficacy and resistance to immune checkpoint therapy. Notably, despite the colorectal cancer focus of this review, this timely information on the IFNy gene signaling pathway is broadly applicable in different types of cancer [8].

Regulatory $\mathrm{T}$ cells (Tregs) are a major immunosuppressive immune cell subset in the tumor microenvironment. Dr. Huppert and her colleagues focus their review on tissue residential Tregs, and their phenotypes and roles in organ specific tumor metastasis. They connect Treg biology to regional immune tolerance, tissue repair, and regeneration. They extend their discussion to immune suppressor capacity of tissue-specific Tregs in the organ/tissuesites of tumor metastasis [9].

Chronic inflammation has been linked to cancer development, progression, and therapy resistance. Given the multifaceted properties of inflammation, Drs. Karin and Shalapour discuss how inflammation triggers epigenetic alterations in cancer cells, fibroblasts, and immune cells in the tumor microenvironment. They pay particular attention to certain specific metabolites, including S-adenosyl methionine (SAM), Acetyl-CoA (Ac-CoA), and $N A D+$, and their roles in histone modification and DNA methylation. As a consequence of this epigenetic regulation, the immune cell states have been altered, causing immune suppression and immune dysfunction [10]. Dr. Rathmell and his colleagues review how tumor cells compete immune cells for key nutrients, as well as the role of the metabolic byproducts of aerobic glycolysis in the regulation of antitumor immunity, and the contribution of tumor glycolysis to immune suppressive mechanisms in the tumor microenvironment. From this perspective, Dr. Rathmell and his colleagues shed light on the development of new classes of agents targeting cancer metabolism [11]. 
In conclusion, this set of reviews gives a much needed overview of the most recent advancement in the field of tumor immunology and its relevance in cancer therapy. We hope that this special issue of CMI represents a meaningful basis to inform, guide, and inspire the development of the next generation of immunotherapeutic regimens for large numbers of cancer patients.

Weiping Zou (iD) ${ }^{1,2}$ 布

${ }^{1}$ Department of Surgery, Center of Excellence for Cancer Immunology and Immunotherapy, University of Michigan Rogel Cancer Center, Ann Arbor, MI, USA. ' Department of Pathology, Graduate Program in Immunology and Graduate Program in Cancer Biology, University of Michigan School of Medicine, Ann Arbor, MI, USA.

凶email: wzou@med.umich.edu

\section{REFERENCES}

1. Zou W. Immunosuppressive networks in the tumor microenvironment and their therapeutic relevance. Nat Rev Cancer 2005;5:263-74. https://doi.org/10.1038/ nrc1586

2. Curiel TJ, Wei S, Dong $H$, Alvarez $X$, Cheng $P$, Mottram $P$, et al. Blockade of B7-H1 improves myeloid dendritic cell-mediated antitumor immunity. Nat Med. 2003;9:562-7. https://doi.org/10.1038/nm863

3. Tang H, Liang Y, Anders RA, Taube JM, Qiu X, Mulgaonkar A, et al. PD-L1 on host cells is essential for PD-L1 blockade-mediated tumor regression. J Clin Invest. 2018;128:580-8. https://doi.org/10.1172/JCl96061

4. Lin H, Wei S, Hurt EM, Green MD, Zhao L, Vatan L, et al. Host expression of PD-L1 determines efficacy of PD-L1 pathway blockade-mediated tumor regression. J Clin Invest. 2018;128:805-15. https://doi.org/10.1172/JCI96113

5. Oh SA, Wu DC, Cheung J, Navarro A, Xiong H, Cubas R, et al. PD-L1 expression by dendritic cells is a key regulator of T-cell immunity in cancer. Nat Cancer. 2020;1:681-91. https://doi.org/10.1038/s43018-020-0075-x

6. Murphy TL, Murphy KM. Dendritic cells in cancer immunology. Cell Mol Immunol. 2022;19:3-13. https://www.nature.com/articles/s41423-021-00741-5
7. Bullock TNJ. CD40 stimulation as a molecular adjuvant for cancer vaccines and other immunotherapies. Cell Mol Immunol. 2022;19:14-22. https://www.nature. com/articles/s41423-021-00734-4

8. Du W, Frankel TL, Green M, Zou WP. IFNy signaling integrity in colorectal cancer immunity and immunotherapy. Cell Mol Immunol. 2022;19:23-32. https://www. nature.com/articles/s41423-021-00735-3

9. Huppert LA, Green MD, Kim L, Chow C, Leyfman Y, Daud Al, et al. Tissue-specifific Tregs in cancer metastasis: opportunities for precision immunotherapy. Cell Mol Immunol. 2022;19:33-45. https://www.nature.com/articles/s41423-021-00742-4.

10. Reinfeld BI, Rathmell WK, Kim TK, Rathmell JC. The therapeutic implications of immunosuppressive tumor aerobic glycolysis. Cell Mol Immunol. 2022;19:46-58. https://www.nature.com/articles/s41423-021-00727-3.

11. Karin M, Shalapour S. Regulation of anti-tumor immunity by inflammationinduced epigenetic alterations. Cell Mol Immunol. 2022;19:59-66. https://www. nature.com/articles/s41423-021-00756-y.

\section{ACKNOWLEDGEMENTS}

This work was supported in part by research grants from the $\mathrm{NIH} / \mathrm{NCl}$ for WZ (CA248430, CA214911, CA123088, CA099985, CA193136, and CA152470) and the $\mathrm{NIH}$ through the University of Michigan Rogel Cancer Center Support Grant (P30CA46592).

\section{COMPETING INTERESTS}

The authors declare no competing interests.

\section{ADDITIONAL INFORMATION}

Correspondence and requests for materials should be addressed to Weiping Zou.

Reprints and permission information is available at http://www.nature.com/reprints 